\title{
Unterschiedliche Wertschätzung, aber gleiche Unterstützungsbereitschaft
}

\section{Soziale Reaktionen auf Optimisten, Pessimisten und Realisten aus der Geberperspektive}

\author{
Manja Vollmann ${ }^{1}$, Britta Renner ${ }^{2}$, Katrin Matiba ${ }^{1}$ und Hannelore Weber ${ }^{1}$ \\ ${ }^{1}$ Universität Greifswald \\ 2Jacobs University Bremen
}

\begin{abstract}
Zusammenfassung. Soziale Netzwerke und insbesondere soziale Unterstützung werden als ein zentraler vermittelnder Mechanismus zwischen Optimismus und Gesundheit diskutiert. In dieser Studie wird die Annahme geprüft, dass Optimisten, Pessimisten und Realisten unterschiedliche soziale Reaktionen hervorrufen. Den Probanden $(N=168)$ wurden Vignetten präsentiert, in denen eine Zielperson (Target) optimistisches, pessimistisches bzw. realistisches Bewältigungsverhalten in einer Stresssituation zeigt. Anschließend wurden per Fragebogen (a) die Bewertung des Verhaltens und der Persönlichkeit des Targets, (b) die Sympathie gegenüber dem Target sowie (c) die Bereitschaft zu sozialer Unterstützung erfasst. Hinsichtlich des Verhaltens, der Persönlichkeit sowie der Sympathie wurden die optimistischen und realistischen Targets positiver bewertet als die pessimistischen Targets. Allerdings gingen diese positiveren Bewertungen der Optimisten und Realisten im Vergleich zu den Pessimisten nicht mit einer höheren Unterstützungsbereitschaft einher. Der Zusammenhang zwischen Optimismus und Gesundheit wird möglicherweise nicht nur über die vom sozialen Umfeld tatsächlich zur Verfügung gestellte Unterstützung, sondern auch durch den Ausdruck sozialer Akzeptanz vermittelt.

Schlüsselwörter: Optimismus, Pessimismus, Realismus, Sympathie, soziale Unterstützung, Geberperspektive
\end{abstract}

Different approval, but equal willingness to provide support: Social responses to optimists, pessimists, and realists from a provider's perspective

\begin{abstract}
It has been presumed that the beneficial health effects of optimism are mediated by social support provided by the social network. This study aims to examine the basic underlying assumption that optimists, pessimists, and realists elicit different social responses. Participants $(N=168)$ were asked to read transcripts of conversations in which optimistic, pessimistic, and realistic targets reported how they were dealing with a stressful situation. Afterwards they completed a questionnaire assessing (a) their evaluation of the target's behavior and personality, (b) their attraction to the target, and (c) their willingness to provide the target with social support. Regarding the evaluation of behavior and personality as well as perceived interpersonal attraction, optimistic and realistic targets were viewed more favorably than pessimistic targets. However, the more positive evaluation of optimists and realists compared to pessimists was not accompanied by a greater willingness to provide them with social support. Thus, the relation between optimism and health may not merely be mediated by social support provided by the network, but also by the expression of social approval.

Key words: optimism, pessimism, realism, social attraction, social support, support provider
\end{abstract}

In zahlreichen Untersuchungen erwies sich Optimismus insbesondere bei der Konfrontation mit belastenden Ereignissen als ein bedeutsamer Prädiktor für das psychische Wohlbefinden und die physische Gesundheit (Überblick: Scheier, Carver \& Bridges, 2001). Soziale Unterstützung wird dabei als ein potenzieller Mediator dieses Zusammenhangs diskutiert (vgl. Peterson \& Bossio, 2001). Es wird angenommen, dass Optimisten im Vergleich zu Pessimisten sozial attraktiver und demzufolge auch besser in soziale Netzwerke integriert sind sowie mehr soziale
Unterstützung von ihrem Umfeld erhalten (z. B. Scheier \& Carver, 1987). Übereinstimmend mit dieser Annahme zeigten sich in verschiedenen Studien positive Zusammenhänge zwischen Optimismus und der Größe des sozialen Netzwerks (Brissette, Scherier \& Carver, 2002; Segerstrom, 2001), der Wahrnehmung von zukünftig verfügbarer Unterstützung (z. B. Symister \& Fried, 2003; Trunzo \& Pinto, 2003) sowie der Einschätzung von Quantität und Qualität bereits erhaltener Unterstützung (Fontaine \& Seal, 1997; Karademas, 2006). Allerdings basieren diese Ergebnisse 
zum Zusammenhang zwischen Optimismus und sozialer Unterstützung auf Einschätzungen des Unterstützungsempfängers. Es stellt sich damit die Frage, ob Optimisten im Vergleich zu Pessimisten tatsächlich mehr Unterstützung zur Verfügung gestellt wird, oder ob sie ihr soziales Umfeld lediglich als unterstützender wahrnehmen, die Einschätzungen also optimistischen Verzerrungen unterliegen.

Hinweise auf eine positive soziale Wirkung von Optimismus finden sich in Arbeiten, die allgemeine soziale Reaktionen aus der Geberperspektive untersucht haben. In experimentellen Untersuchungen wurde gezeigt, dass Personen mit optimistischen Zukunftserwartungen bzw. optimistisch verzerrten Risikoeinschätzungen mehr soziale Anerkennung erfahren als Personen mit pessimistischen Zukunftserwartungen bzw. pessimistisch verzerrten Risikoeinschätzungen (Carver, Kus \& Scheier, 1994; HelwegLarson, Sadeghian \& Webb, 2002). Weiterhin wird Personen mit positiven im Vergleich zu negativen Selbstbewertungen mehr Sympathie entgegengebracht (z. B. Robinson, Johnson \& Shields, 1995; Silver, Wortman \& Crofton, 1990). Ferner geht eine positive Selbstdarstellung mit einer stärkeren Beliebtheit bei Freunden und positiveren Persönlichkeitsbewertungen einher (Taylor, Lerner, Sherman, Sage \& McDowell, 2003). Demnach ist die generelle soziale Resonanz auf Optimisten im Vergleich zu Pessimisten positiver, so dass die Annahme naheliegt, dass Optimisten auch mehr soziale Unterstützung zur Verfügung gestellt wird. Damit würde die Einschätzung von Optimisten, mehr soziale Unterstützung zu erhalten, keine optimistische Verzerrung, sondern eine akkurate Abbildung der tatsächlich erbrachten Unterstützungsleistungen des sozialen Umfeldes darstellen.

Allerdings zeigen zwei neuere Studien, die direkt den Zusammenhang zwischen dem Optimismus des Unterstützungsempfängers (Target) und verschiedenen sozialen Reaktionen aus der Geberperspektive untersuchten, ein komplexeres Zusammenhangsmuster. In diesen Untersuchungen wurde erstmalig ein breites Spektrum an sozialen Reaktionen erfasst (Vollmann, Renner \& Weber, 2007). Neben Einschätzungen bezüglich der Angemessenheit und Effektivität des Verhaltens des Targets wurden auch die Bewertung der Persönlichkeit, die entgegengebrachte Sympathie sowie die Bereitschaft zu sozialer Unterstützung erfasst. Darüber hinaus wurden die Targets auf der Grundlage von prototypischen Verhaltensmustern konstruiert, die Optimisten, Pessimisten und Realisten bei der Bewältigung unterschiedlich kontrollierbarer Stresssituationen zeigen. Diese prototypischen Verhaltensmuster repräsentieren soziale Konzeptionen von Optimismus, Pessimismus und Realismus und umfassen Gedanken, Gefühle, Ziele und Handlungen, die anhand einer modifizierten Version des Act Frequency Ansatzes gewonnen wurden (vgl. Weber, Vollmann \& Renner, 2007).

Die Ergebnisse der Studien von Vollmann et al. (2007) zeigten wie erwartet, dass Optimisten und Realisten hinsichtlich ihres Verhaltens und ihrer Persönlichkeit positiver bewertet wurden als Pessimisten. Ihr Verhaltensmus- ter wurde als angemessener und effektiver beurteilt und ihre Persönlichkeit wurde positiver eingeschätzt als die der Pessimisten. Weiterhin wurden Optimisten und Realisten auch als sympathischer wahrgenommen als Pessimisten. Überraschenderweise zeigten sich jedoch keine Unterschiede hinsichtlich der Unterstützungsbereitschaft. Die Untersuchungsteilnehmer waren in gleicher Weise bereit, Optimisten, Realisten und Pessimisten zu unterstützen. Diese vergleichbar hohe Unterstützungsbereitschaft zeigte sich sowohl in kontrollierbaren als auch unkontrollierbaren Stresssituationen sowie in Stresssituationen aus unterschiedlichen Lebensbereichen. Die Befunde legen nahe, dass der von Optimisten im Vergleich zu Pessimisten berichtete Erhalt von mehr Unterstützung eine unrealistisch verzerrte Wahrnehmung darstellt, der nicht mit Unterschieden in den tatsächlich erbrachten Unterstützungsleistungen des sozialen Umfeldes übereinstimmt. Diese Befunde stehen damit in einem gewissen Widerspruch zu aktuellen theoretischen Annahmen und verweisen in Abhängigkeit von der Art der sozialen Reaktion auf unterschiedliche Reaktionsmuster gegenüber Optimisten und Pessimisten. Möglicherweise werden Optimisten zwar mehr sozial geschätzt als Pessimisten, aber nicht zwangsläufig auch mehr sozial unterstützt. Es stellt sich jedoch die Frage, inwieweit diese Ergebnisse generalisierbar sind.

\section{Die vorliegende Studie}

Ein wichtiger Aspekt bei der Frage der Generalisierbarkeit ist die Art der Präsentation der Informationen über die potenziellen Unterstützungsempfänger. In den beiden Studien von Vollmann et al. (2007) wurden die Informationen in Form eines komplexen Verhaltensmusters über Audioaufnahmen präsentiert. Diese Audioaufnahmen umfassten eine Unterhaltung, in der das Target gegenüber einer Gesprächspartnerin entweder prototypisch optimistische, realistische oder pessimistische Gedanken, Gefühle, Ziele und Verhaltensweisen im Umgang mit einer belastenden Situation zum Ausdruck brachte. Es handelte sich demnach um komplexere Informationen als in den meisten bisherigen Untersuchungen. Eine mögliche Erklärung dafür, dass die Versuchsteilnehmer keine differenzielle Unterstützungsbereitschaft gegenüber Optimisten, Realisten und Pessimisten gezeigt haben, könnte daher darin liegen, dass sie aufgrund des Audiomodus und der Tatsache, dass sie die Unterhaltung nur einmal hörten, die Informationen eher oberflächlich und intuitiv verarbeitet haben. Schriftliche Vignetten, wie sie z. B. in der Arbeit von Carver et al. (1994) verwendet wurden, ermöglichen hingegen eine von den Teilnehmern selbstgesteuerte und damit möglicherweise tiefere Verarbeitung der Informationen. Um den Einfluss des Präsentationsmodus zu untersuchen, wurden in der vorliegenden Studie die prototypischen Verhaltensmuster von Optimisten, Pessimisten und Realisten im Umgang mit einer belastenden Beziehungssituation in Form von Vignetten dargeboten.

Wie in den Studien von Vollmann et al. (2007) wurden die prototypischen Verhaltensmuster von Optimisten, 
Realisten und Pessimisten sowohl in einer kontrollierbaren als auch einer unkontrollierbaren Belastungssituation präsentiert, da in Abhängigkeit von der Kontrollierbarkeit der Situation unterschiedliches Bewältigungsverhalten als effektiv erachtet wird und somit eine Anpassung des Verhaltens an die situationalen Gegebenheiten erforderlich ist (Folkman \& Moskowitz, 2004; Weber, 2003). Die präsentierten Verhaltensmuster wurden in den Studien von Weber et al. (2007) auf der Grundlage sozialer Konzeptionen von Optimismus, Pessimismus und Realismus ermittelt. Demnach zeichnen sich Pessimisten aus der Sicht von sozialen Urteilern sowohl in kontrollierbaren als auch unkontrollierbaren Situationen durch ein eher rigides und dysfunktionales Verhaltensmuster aus. Optimisten hingegen wird ein teilweise adaptives Verhaltensmuster zugeschrieben, in dem zwar die Gedanken und Gefühle an die Kontrollierbarkeit der Situation angepasst werden, jedoch nicht die Ziele und das Verhalten,während Realisten durch eine vollständige Anpassung des Verhaltensmusters gekennzeichnet sind (vgl. Weber et al., 2007).

In der vorliegenden Untersuchung sollte aus der Geberperspektive untersucht werden, (1) ob Optimisten im Vergleich zu Pessimisten unabhängig von der Kontrollierbarkeit der Situation positivere Bewertungen hinsichtlich ihres Verhaltens, ihrer Persönlichkeit sowie mehr Sympathie hervorrufen und mehr Unterstützungsbereitschaft auslösen und (2) ob Optimisten und Realisten in kontrollierbaren Situationen gleichermaßen positive Reaktionen auslösen, während in unkontrollierbaren Situationen Realisten auf Grund ihres adaptiveren Verhaltensmusters positivere Reaktionen hervorrufen als Optimisten.

\section{Methode}

\section{Stichprobe}

An der Untersuchung nahmen 168 Studierende (50\% Frauen) verschiedener Fachrichtungen der Universität Greifswald teil. Das mittlere Alter betrug 22 Jahre $(S D=$ 2.77), wobei die jüngste Versuchsperson 18 Jahre alt war und die älteste 34 Jahre.

\section{Ablauf der Untersuchung}

Die Studie umfasste 12 verschiedene Vignetten, die auf der Grundlage eines 3 „Verhaltensmuster des Targets“ (Optimist vs. Pessimist vs. Realist) $\times 2$ „Kontrollierbarkeit“ (kontrollierbar vs. unkontrollierbar) $\times 2$,Geschlecht des Targets“ (männlich vs. weiblich) Designs konstruiert wurden. Das Geschlecht der Probanden wurde ausbalanciert und ihre Zuweisung zu einer der 12 Bedingungen erfolgte nach Zufall.

Die Probanden wurden darüber informiert, dass die Studie zum Thema Wahrnehmung und Einschätzung von Personen durchgeführt werde. Sie erhielten einen Fragebogen, in dem sie zunächst gebeten wurden, ein kurzes
Gespräch zwischen zwei Mitbewohnern aufmerksam zu lesen und sich einen Eindruck vom Target zu bilden. Anschließend wurden (a) die Bewertung des Verhaltens und der Persönlichkeit des Targets, (b) die Sympathie gegenüber dem Target sowie (c) die Bereitschaft zu sozialer Unterstützung erhoben.

\section{Stimulusmaterial}

Das Stimulusmaterial bestand aus Vignetten mit einem kurzen Gespräch zwischen zwei Mitbewohnern, in dem der/die potenzielle Unterstützungsempfänger/in (Target) entweder ein optimistisches, pessimistisches oder realistisches Verhaltensmuster als Reaktion auf eine kontrollierbare bzw. unkontrollierbare Stresssituation zeigt. In beiden Situationen erzählt das Target, dass er/sie sich verliebt und es der betreffenden Person auch gesagt hat. In der kontrollierbaren Situation wurde das Target zurückgewiesen, da die andere Person ihn/sie erst besser kennen lernen möchte. In der unkontrollierbaren Situation wurde das Target zurückgewiesen, da die andere Person seine/ ihre Gefühle nicht erwidert. ${ }^{1}$ Die Vignetten wurden auf der Grundlage des Audiomaterials von Vollmann et al. (2007) adaptiert, um eine möglichst hohe Vergleichbarkeit des Inhalts zu gewährleisten.

Das Gespräch zwischen dem Target und der Mitbewohnerin ereignete sich während einer gemeinsamen Kaffeepause. Im Verlaufe des Gesprächs kommt die Mitbewohnerin auf die Stresssituation zu sprechen, indem sie das Target fragt: „Sag mal, hast Du nicht neulich erzählt, dass Du jemanden kennen gelernt hast, den Du sehr sympathisch findest? Was ist eigentlich aus der Sache geworden?" Daraufhin berichtet das Target über die Entgegnung der betreffenden Person und wird von der Mitbewohnerin durch Zwischenfragen wie „Was denkst Du darüber?“ und „Was hast Du jetzt vor?“ ermutigt, über seine/ihre Gedanken, Gefühle, Ziele und geplanten Handlungen zu sprechen. Die in Erwiderung auf diese Fragen berichteten optimistischen, pessimistischen bzw. realistischen Reaktionen des Targets basieren auf den affektivkognitiven Verhaltensmustern, die in früheren Studien als prototypisch für Optimisten, Pessimisten und Realisten im Umgang mit den zwei Stresssituationen identifiziert wurden (Weber et al., 2007). Ausschnitte aus den Gesprächen für die kontrollierbare Situation sind im Anhang dargestellt.

Das optimistische Verhaltensmuster zeichnet sich in der kontrollierbaren Situation durch eine positive Konstruktion der Situation, positive Ergebniserwartungen, positive Gefühle, die Weiterverfolgung des Ziels und aktive Zielerreichungsversuche aus. In der unkontrollierbaren Situation ist das optimistische Verhaltensmuster durch positive Ergebniserwartungen, optimistische Attributio-

\footnotetext{
1 In einer früheren Studie wurden die beiden Situationen als mittelmäßig belastend und als signifikant verschieden in ihrer Kontrollierbarkeit eingeschätzt (Weber et al., 2007).
} 
nen, positive als auch negative Gefühle, Persistenz in der Zielweiterverfolgung und aktive Zielerreichungsversuche gekennzeichnet. Das pessimistische Verhaltensmuster besteht in beiden Situationen aus einer negativen Konstruktion der Situation, negativen Ergebniserwartungen, pessimistischen Attributionen, negativen Gefühlen, der Aufgabe des Ziels, Rückzug sowie dem Ausdruck von und der Fokussierung auf die erlebten negativen Emotionen. Weiterhin beinhalten die pessimistischen Verhaltensmuster negative selbstbezogene Kognitionen und Emotionen, wie Selbstbeschuldigung, Selbstmitleid und Schuldgefühle. Das realistische Verhaltensmuster ist in der kontrollierbaren Situation durch Akzeptanz der Situation, weder positive noch negative Gefühle, eine verhaltene Weiterverfolgung des Ziels sowie Affektkontrolle charakterisiert. In der unkontrollierbaren Situation zeichnet sich das realistische Verhaltensmuster durch die Akzeptanz der Situation, positive als auch negative Gefühle, die Aufgabe des Ziels und Versuche der Affektkontrolle aus (vgl. Weber et al., 2007).

Die Vignetten wurden mit einem männlichen und einem weiblichen Target präsentiert, um den möglichen Einfluss des Geschlechts zu kontrollieren.

\section{Erhebungsinstrumente}

Bewertung des Verhaltens. Das Verhalten des Targets wurde in Bezug auf die Angemessenheit und die Effektivität mit jeweils zwei Items bewertet. Die Einschätzungen erfolgten auf einer 7-stufigen Skala von 1 (gar nicht) bis 7 (sehr). Zur Erfassung der Angemessenheit gaben die Probanden an, wie angemessen und wie akzeptabel das Verhalten des Targets war (Korrelation zwischen den beiden Items $r=.71$ ). Die Effektivität wurde über die Bewertung erhoben, wie effektiv und wie zielführend das Verhalten des Targets war $(r=.70)$. Für die Angemessenheit und die Effektivität wurde durch Mittelung der zwei Items jeweils ein Gesamtwert errechnet.

Persönlichkeitsbewertung. Die Bewertung der Persönlichkeit des Targets erfolgte durch sechs Items, die je zur Hälfte die Zuschreibung von Erfolg (z. B. „Die Person wird alle ihre Ziele erreichen“; adaptiert nach Montoya \& Horton, 2004) sowie die Zuschreibung von sozialer Anerkennung (z. B. „Die Person ist sozial gut integriert“) erfassen. Die Beantwortung erfolgte auf einer 7-stufigen Skala von 1 (stimme nicht zu) bis 7 (stimme sehr zu). Zur Berechnung eines Gesamtwertes wurden die Itemantworten gemittelt $(\alpha=$.89).

Sympathie. Die dem Target entgegengebrachte Sympathie wurde mit sieben aus vorliegenden Studien adaptierten Items erhoben (Carver et al., 1994; Helweg-Larsen et al., 2002; Silver et al., 1990). Die Skala beinhaltet zwei Items, die die affektive Komponente erfassen (z. B. „Ich würde die Person mögen, wenn ich sie treffen würde“) und fünf Items, die die behaviorale Komponente erfassen (z. B. „Ich würde mit der Person gerne zu einer Studentenparty gehen“). Zur Beurteilung der Items stand den Probanden eine 7-stufige Skala von 1 (mit Sicherheit nicht) bis 7 (mit Sicherheit ja) zur Verfügung. Der Sympathiewert wurde durch die Mittelung der Itemantworten berechnet ( $\alpha=$ $.92)$.

Unterstützungsbereitschaft. Die Unterstützungsbereitschaft wurde mit fünf Items erfasst, die der Studie von Schwarzer und Weiner (1990) entnommen wurden. Auf einer 7-stufigen Skala von 1 (mit Sicherheit nicht) bis 7 (mit Sicherheit ja) gaben die Probanden an, inwieweit sie bereit wären, dem Target soziale Unterstützung zukommen zu lassen (z. B. „Ich würde der Person mit Rat und Tat zur Seite stehen"). Die Itemantworten wurden zu einem Gesamtwert zusammengefasst $(\alpha=.90)$.

Kontrollvariablen. Für einen Manipulation Check gaben die Probanden auf einer 7-stufigen Skala von 1 (gar nicht) bis 7 (sehr) an, wie optimistisch, pessimistisch und realistisch sie das Verhalten des Targets einschätzen.

\section{Ergebnisse}

\section{Manipulation Check}

Zur Überprüfung der experimentellen Manipulation wurde für jede der drei Kontrollvariablen eine Varianzanalyse mit dem Faktor „Verhaltensmuster des Targets“ gerechnet. Die optimistischen Targets $(M=6.23, S D=1.04)$ wurden als signifikant optimistischer eingeschätzt als die pessimistischen und die realistischen Targets $(M=1.13, S D=$ 0.33 und $M=3.71, S D=1.85), F(2,165)=237.66, p<.001$, $\eta^{2}=.74$, Bonferroni-Tests, $p \mathrm{~s}<.001, d s>2$. Weiterhin wurden die pessimistischen Targets $(M=6.38, S D=1.32)$ als signifikant pessimistischer bewertet als die optimistischen und die realistischen Targets $(M=1.70, S D=1.11$ und $M=3.46, S D=1.73), F(2,165)=157.76, p<.001, \eta^{2}=$ .66 , Bonferroni-Tests, $p s<.001, d s>1.8$. Ebenso wurden die realistischen Targets $(M=5.41, S D=1.32)$ als signifikant realistischer wahrgenommen als die optimistischen und die pessimistischen Targets $(M=3.71, S D=1.56$ und $M=3.50, S D=1.65), F(2,165)=26.72, p<.001, \eta^{2}=.25$, Bonferroni-Tests, $p s<.001, d s>1$. Der Manipulation Check bestätigt, dass sich die drei Verhaltensmuster wie erwartet deutlich voneinander unterscheiden.

\section{Analysen}

Zur Analyse der sozialen Reaktionen auf die optimistischen, realistischen und pessimistischen Targets wurde zunächst über alle abhängigen Variablen hinweg eine 3 „Verhaltensmuster des Targets“ $\times 2$, ,Kontrollierbarkeit“ $\times$ 2 „Geschlecht des Targets“ multivariate Varianzanalyse gerechnet. Für den Faktor „Geschlecht des Targets“ ergaben sich keine signifikanten Haupt- oder Interaktionseffekte, Fs $<2$, ns. Dieser Faktor wurde deshalb in allen folgenden Analysen nicht weiter berücksichtigt. Zur weiteren Datenanalyse wurden 3 ,Verhaltensmuster des Targets“ $\times 2$ „Kontrollierbarkeit“ Varianzanalysen durchge- 


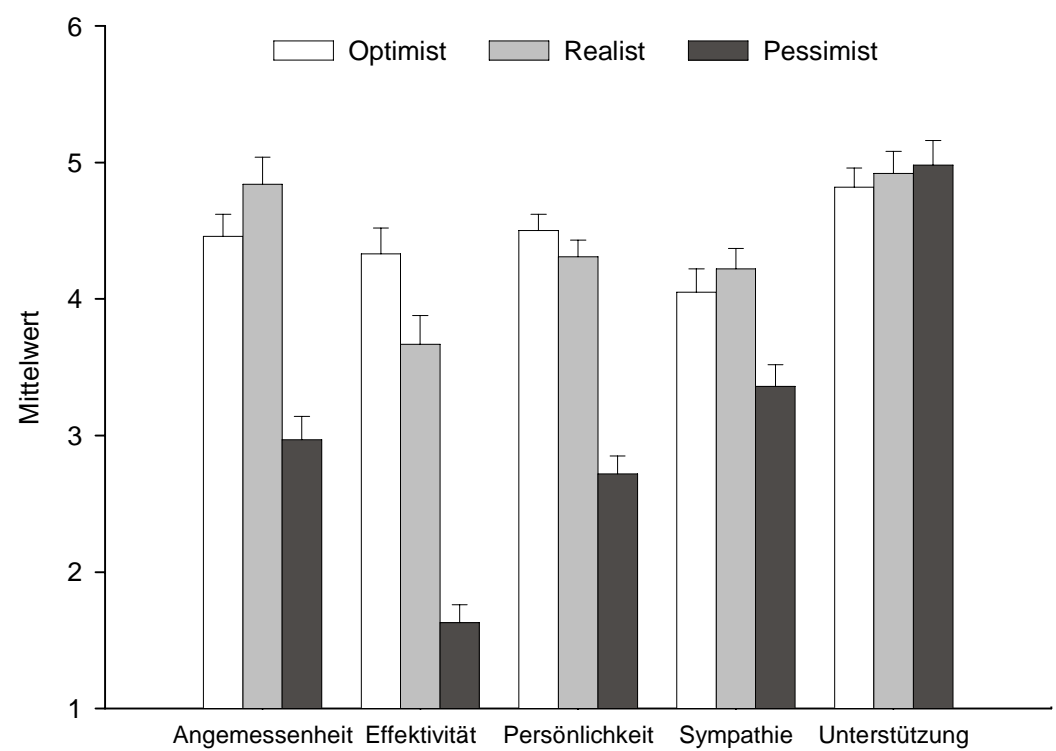

Abbildung 1. Mittelwerte (mit Standardfehlern) der sozialen Reaktionen in Abhängigkeit vom gezeigten Verhaltensmuster (höhere Werte stehen für eine positivere Bewertung).

führt. Signifikante Haupteffekte wurden mit bonferroniadjustierten Mehrfachvergleichen analysiert und signifikante Interaktionen wurden durch die Analyse der einfachen Haupteffekte auf den Stufen des Faktors „Kontrollierbarkeit“" spezifiziert. Die Ergebnisse sind zusammenfassend in Abbildung 1 dargestellt.

\section{Bewertung des Verhaltens}

Die Varianzanalyse der Angemessenheitsbewertung des Verhaltens des Targets erbrachte einen signifikanten Haupteffekt für den Faktor „Verhaltensmuster des Targets“, $F(2,162)=30.23, p<.001, \eta^{2}=.27$. Sowohl die optimistischen als auch die realistischen Verhaltensmuster $(M=4.46, S D=1.17$ und $M=4.84, S D=1.49)$ wurden als signifikant angemessener bewertet als die pessimistischen Verhaltensmuster $(M=2.97, S D=1.31), p s<.001, d s$ $>$ 1.3. Wie die Abbildung 1 und die hohe Effektstärke zeigt, wurde das Verhalten von Optimisten und Realisten als deutlich angemessener beurteilt als das der Pessimisten. Alle anderen Effekte wurden nicht signifikant, $F \mathrm{~s}<1$, $n s$.

Bezüglich der Effektivität des Verhaltens des Targets ergab die Varianzanalyse ebenfalls einen signifikanten Haupteffekt für den Faktor „Verhaltensmuster des Targets“, $F(2,162)=60.54, p<.001, \eta^{2}=.43$. Hier zeigte sich ein noch deutlicherer Unterschied zwischen den Bewertungen der optimistischen und realistischen Verhaltensmuster auf der einen Seite und den pessimistischen Verhaltensmustern auf der anderen Seite (vgl. Abbildung 1). Die pessimistischen Verhaltensmuster wurden als nur im geringen Maße effektiv beurteilt $(M=1.63, S D=1.00)$, während den realistischen $(M=3.67, S D=1.60)$ und den optimistischen $(M=4.33, S D=1.42)$ Verhaltensmustern eine deutlich höhere Effektivität zugesprochen wurde, $p s$
$<001, d s>1.5$. Die Kontrastanalysen zeigten darüber hinaus, dass die optimistischen Verhaltensmuster als signifikant effektiver bewertet wurden als die realistischen Verhaltensmuster, $p<.05, d=0.5$. Dieser Unterschied zeichnet sich jedoch durch eine deutlich geringere Effektstärke aus. Weitere Effekte wurden nicht signifikant, $F_{\mathrm{s}}<3$, ns.

\section{Persönlichkeitsbewertung}

In Bezug auf die Bewertung der Persönlichkeit des Targets ergab sich ein ähnliches Befundmuster. Die Varianzanalyse erbrachte wiederum einen signifikanten Haupteffekt für den Faktor „Verhaltensmuster des Targets“, $F(2,162)=62.20, p<.001, \eta^{2}=.43$. Die Persönlichkeit von optimistischen als auch von realistischen Targets $(M=4.50$, $S D=0.89$ und $M=4.31, S D=0.93$ ) wurde deutlich positiver bewertet als die der pessimistischen Targets $(M=2.72, S D=0.95)$, $p s<.001, d s>1.7$. Demnach wurden die optimistischen und die realistischen Targets als erfolgreicher und sozial akzeptierter eingeschätzt als die pessimistischen Targets. Auch dieser Unterschied zeichnet sich durch eine hohe Effektstärke aus. Alle weiteren Effekte wurden nicht signifikant, Fs $<1$, ns.

\section{Sympathie}

Die Varianzanalyse bezüglich der Sympathie gegenüber dem Target erbrachte einen signifikanten Haupteffekt für den Faktor „Verhaltensmuster des Targets“, $F(2,162)=$ 8.13, $p<.001, \eta^{2}=.09$. Sowohl die optimistischen als auch die realistischen Targets $(M=4.05, S D=1.27$ und $M=4.22$, $S D=1.16$ ) wurden als signifikant sympathischer wahrgenommen als die pessimistischen Targets $(M=3.36, S D=$ $1.20)$, $p s<.01, d s>0.5$. Kein anderer Effekt wurde signifikant, $F_{\mathrm{S}}<2$, ns.

\section{Unterstützungsbereitschaft}

In Bezug auf die Unterstützungsbereitschaft erbrachte die Varianzanalyse keine signifikanten Haupteffekte oder Interaktionen, $F s<2$, ns. Wie die Abbildung 1 zeigt, waren die Probanden im vergleichbar hohen Maß bereit, Optimisten, Realisten und Pessimisten zu unterstützen.

\section{Diskussion}

Die vorliegende Studie untersuchte aus einer Geberperspektive verschiedene soziale Reaktionen auf Optimisten, Realisten und Pessimisten, die sich in zwei unterschiedlich kontrollierbaren Belastungssituationen befanden. Die Befunde zeigten für diese beiden Belastungssituationen ein im hohen Maße konsistentes Muster. 


\section{Optimisten vs. Pessimisten}

Generell waren die sozialen Reaktionen gegenüber Optimisten erheblich positiver als gegenüber Pessimisten. Optimisten wurden im Vergleich zu Pessimisten hinsichtlich der Angemessenheit und Effektivität ihres Verhaltens sowie ihrer Persönlichkeit wesentlich positiver bewertet. Zudem lösten sie auch positivere affektive Reaktionen aus, denn sie wurden als signifikant sympathischer wahrgenommen. Trotz dieser ausgeprägten positiveren Reaktionen fanden sich wie auch bei Vollmann et al. (2007) keine Unterschiede in der Bereitschaft, Optimisten und Pessimisten soziale Unterstützung zukommen zu lassen. Beide lösten ein vergleichbar hohes Maß an Unterstützungsbereitschaft aus. Damit konnte auf Grundlage der schriftlichen Vignetten das Ergebnismuster der auf Audioaufnahmen basierenden Studien von Vollmann et al. (2007) vollständig repliziert werden. Dies ist von hoher Bedeutsamkeit, da Vignetten theoretisch eher eine selbstgesteuerte und damit tiefere Verarbeitung ermöglichen, während Audioaufnahmen eine mehr intuitive und oberflächliche Verarbeitung induzieren. Darüber hinaus vermitteln $\mathrm{Au}-$ dioaufnahmen zusätzlich nonverbale emotionale Informationen durch die Stimmmodulation des Targets, was wiederum die Reaktionen auf der Geberseite systematisch beeinflussen könnte. Gemäß den vorliegenden Ergebnissen hat der Präsentationsmodus jedoch keinen entscheidenden Einfluss auf die sozialen Reaktionen der potenziellen Unterstützungsgeber. Die Replikation des differenziellen Reaktionsmusters auf der Geberseite in Abhängigkeit von der Art der sozialen Reaktion mit Hilfe einer anderen Methode und Stichprobe verweist auf die Generalisierbarkeit des Befundmusters.

Diesen Befunden zufolge ist der von Optimisten häufig berichtete Erhalt von mehr sozialer Unterstützung möglicherweise eine optimistisch verzerrte Wahrnehmung, die nicht die tatsächlichen Unterstützungsleistungen des sozialen Netzwerkes abbildet. Wenn Optimisten allerdings nicht mehr Unterstützung gegeben wird als Pessimisten, stellt sich die Frage, wie dies mit theoretischen Annahmen und früheren Befunden in Einklang gebracht werden kann, nach denen soziale Unterstützung die Beziehung zwischen Optimismus und Gesundheit mediiert (z. B. Shen, McCreary \& Myers, 2004; Trunzo \& Pinto, 2003). Die vorliegende Studie hat gezeigt, dass Optimisten in Bezug auf ihr Verhalten, ihre Persönlichkeit sowie ihre Sympathie in der Tat deutlich positiver bewertet wurden als Pessimisten. Eine mögliche Erklärung könnte daher sein, dass Optimisten diese positiveren Reaktionen bereits als Unterstützung interpretieren und dementsprechend auch angeben, mehr soziale Unterstützung zu erhalten. Eine andere Erklärungsmöglichkeit ist, dass Optimisten dazu neigen, die positiven sozialen Reaktionen zu generalisieren und folglich mehr potenziell verfügbare Unterstützung wahrnehmen, was wiederum positive Auswirkungen auf die Gesundheit hat. Demnach würde vornehmlich die wahrgenommene Verfügbarkeit von Unterstützung und weniger die tatsächlich zur Verfügung gestellte Unterstützung die vermittelnde Variable darstellen. Dies wäre vereinbar mit Studien, die gezeigt haben, dass vor allem die wahrgenommene und nicht so sehr die bereitgestellte Unterstützung positive Auswirkungen auf Wohlbefinden und Stresserleben hat (vgl. Helgeson, 2003).

Alternativ könnte die gleichermaßen hohe Bereitschaft, Optimisten und Pessimisten zu unterstützen, durch die Art der Erfassung erklärt werden. Die eingesetzte Skala erfasst die anfängliche Bereitschaft zu sozialer Unterstützung, die sich jedoch über die Zeit hinweg verändern könnte (z. B. Dunkel-Schetter \& Bennett, 1990), insbesondere wenn Aufwand und Kosten von Unterstützungsleistungen erkennbar werden. Diese Interpretation stünde im Einklang mit Befunden, nach denen depressive Personen vornehmlich in langfristigen Beziehungen und bei konstant negativer Stimmungslage soziale Ablehnung erfahren (Marcus \& Nardone, 1992; Winer, Bonner, Blaney \& Murray, 1981). Demnach könnte die Bereitschaft, Pessimisten zu unterstützen über die Zeit hinweg abnehmen, da das dysfunktionale Verhaltensmuster der Pessimisten keine Verbesserung der Situation erwarten lässt und der Ausdruck negativer Gefühle zunehmend emotional belastend für den Unterstützungsgeber sein kann. Unterschiede bezüglich der Unterstützungsbereitschaft gegenüber Optimisten und Pessimisten würden dann erst nach mehrfachen Interaktionen sichtbar werden.

Eine weitere Erklärung für die uniform hohe Unterstützungsbereitschaft könnte darin bestehen, dass die eingesetzte Skala nicht zwischen verschiedenen Arten sozialer Unterstützung, wie emotionaler, instrumenteller und informationeller Unterstützung, differenziert (vgl. Helgeson, 2003). Möglicherweise passen jedoch Unterstützungsgeber die Art ihrer Unterstützungsleistungen an die Bedürfnisse des Empfängers an, so dass mögliche Interaktionen mit einer globalen Messung der sozialen Unterstützungsbereitschaft nicht aufgedeckt werden konnten. So wäre es denkbar, dass Pessimisten vor allem emotionale Unterstützung gegeben wird, um ihre negative Stimmung abzumildern, während Optimisten insbesondere instrumentell bei ihren Zielerreichungsversuchen unterstützt werden. Gemittelt über die verschiedenen Arten sozialer Unterstützung ergäben sich demnach keine Unterschiede in der Bereitschaft zu sozialer Unterstützung gegenüber Optimisten und Pessimisten. Weiterhin könnten Erwartungen und Persönlichkeitsmerkmale auf Seiten des Gebers die Bereitschaft zu sozialer Unterstützung beeinflussen.

\section{Optimisten vs. Realisten}

Die Befunde zeigten keine prägnanten Unterschiede zwischen den sozialen Reaktionen gegenüber Optimisten und Realisten. Unabhängig von der Adaptivität des Verhaltensmusters in Bezug auf die Kontrollierbarkeit der Situation wurden Optimisten und Realisten generell positiv beurteilt. Optimisten und Realisten wurden in Bezug auf die Angemessenheit ihres Verhaltens und ihre Persönlichkeit gleichermaßen positiv bewertet. Darüber hinaus wurden sie im gleichen Maße als sympathisch wahrgenommen und die Probanden waren gleichermaßen bereit, ihnen eine hohe soziale Unterstützung zukommen zu lassen. Das Ver- 
halten der Optimisten wurde lediglich als etwas effektiver bewertet. Bemerkenswert ist, dass die Ergebnisse nahe legen, dass Optimisten im Vergleich zu Realisten auch bei unzureichender Anpassung an situative Gegebenheiten wie durch positive Ergebniserwartungen und persistente Zielverfolgung in der unkontrollierbaren Situation - nicht zwangsläufig negativere soziale Reaktionen hervorrufen. Ein Grund für die gleichermaßen positiven Reaktionen auch in der unkontrollierbaren Situation könnte sein, dass die erwarteten Unterschiede in den Reaktionen auch hier zeitverzögert hervortreten, nämlich dann, wenn die Diskrepanz zwischen dem Verhaltensmuster und den situationalen Bedingungen deutlich wird (vgl. Paulhus, 1998). Eventuell rufen Optimisten erst dann ausgeprägte negative Reaktionen hervor, wenn nach mehrfachen erfolglosen Anstrengungen keine Anpassung der Situationsbewertung und der Zielsetzung an die situativen Bedingungen erfolgt.

\section{Einschränkungen}

In der vorliegenden Studie wurden die sozialen Reaktionen auf schriftlich präsentierte Verhaltensmuster in Form von Selbstberichten erfasst. Im Vergleich zu tatsächlichen Interaktionssituationen birgt dieses Vorgehen die Gefahr, dass die Antworten durch soziale Erwünschtheit oder die Befolgung sozialer Normen beeinflusst werden. Außerdem wurde die Bereitschaft zu sozialer Unterstützung erfasst und nicht tatsächlich erbrachte Unterstützungsleistungen. Es erscheint plausibel, dass die Intention einen Prädiktor für das Verhalten darstellt (vgl. Ajzen \& Madden, 1986), jedoch bleibt offen, in welchem Ausmaß tatsächliche Unterstützungsleistungen für Optimisten, Pessimisten und Realisten erbracht werden. In weiteren Studien sollten daher experimentelle Anordnungen mit realen Interaktionssituationen genutzt werden, in denen das aktuelle Unterstützungsverhalten direkt beobachtbar und erfassbar ist.

Da die vorliegenden Ergebnisse auf einer studentischen Stichprobe basieren, wäre es zudem sinnvoll, die Generalisierbarkeit der Ergebnisse auf andere Personengruppen zu untersuchen. Möglicherweise haben ältere Personen oder Personen mit einem anderen kulturellen Hintergrund andere Vorstellungen von Optimismus, Pessimismus und Realismus sowie andere Verhaltensnormen hinsichtlich der Bereitstellung sozialer Unterstützung, was dann wiederum zu anderen sozialen Reaktionen führt. In weiteren Untersuchungen sollte zudem geprüft werden, inwieweit die Ergebnisse auf andere relevante Lebensbereiche, wie zum Beispiel gesundheitsbezogene Stresssituationen, generalisiert werden können.

Weiterhin wurden die sozialen Reaktionen der Probanden auf ihnen unbekannte Personen erfasst. Da Aspekte interpersoneller Beziehungen, wie die Länge oder vorherige Austauschprozesse, einen Einfluss auf emotionale Reaktionen und die Bereitschaft zu sozialer Unterstützung haben können (z. B. Marcus \& Nardone, 1992; Winer et al., 1981), sind die Ergebnisse möglicherweise nur begrenzt auf länger andauernde Beziehungen übertragbar. In weiteren Studien wäre es deshalb interessant, soziale Reaktionen auf Optimisten, Pessimisten und Realisten in bereits bestehenden Beziehungen, beispielsweise bei Ehepaaren oder Freunden, zu untersuchen.

\section{Schlussfolgerungen}

In der vorliegenden Studie konnten in Übereinstimmung mit den Studien von Vollmann et al. (2007) zwar deutlich positivere generelle soziale Reaktionen auf Optimisten und Realisten im Vergleich zu Pessimisten gezeigt werden, aber keine Unterschiede in der Unterstützungsbereitschaft. Dies legt die Vermutung nahe, dass Optimisten diese positiven Akzeptanzreaktionen generalisieren und deshalb mehr Unterstützung wahrnehmen. Demnach wäre der Zusammenhang zwischen Optimismus und Gesundheit nicht in erster Linie durch das Ausmaß an tatsächlich erbrachten Unterstützungsleistungen des sozialen Netzwerkes, sondern vielmehr durch die wahrgenommene soziale Anerkennung vermittelt. Weiterhin legen die Ergebnisse nahe, dass Optimismus im Vergleich zu Realismus auch bei stärkeren Verzerrungen der Realität zumindest in der ersten Reaktion keine negativen sozialen Konsequenzen zur Folge hat.

\section{Literatur}

Ajzen, I. \& Madden, T. J. (1986). Prediction of goal-directed behavior: Attitudes, intentions, and perceived behavioral control. Journal of Experimental Social Psychology, 22, 453-474.

Brissette, I., Scheier, M. F. \& Carver, C. S. (2002). The role of optimism in social network development, coping, and psychological adjustment during a life transition. Journal of Personality and Social Psychology, 82, 102-111.

Carver, C. S., Kus, L. A. \& Scheier, M. F. (1994). Effects of good versus bad mood and optimistic versus pessimistic outlook on social acceptance versus rejection. Journal of Social and Clinical Psychology, 13, 138-151.

Folkman, S. \& Moskowitz, J. T. (2004). Coping: Pitfalls and promise. Annual Review of Psychology, 55, 745-774.

Fontaine, K. R. \& Seal, A. (1997). Optimism, social support, and premenstrual dysphoria. Journal of Clinical Psychology, 53, 243-247.

Helgeson, V. S. (2003). Social support and quality of life. Quality of Life Research: An International Journal of Quality of Life Aspects of Treatment, Care and Rehabilitation, 12, 2531

Helweg-Larsen, M., Sadeghian, P. \& Webb, M. S. (2002). The stigma of being pessimistically biased. Journal of Social and Clinical Psychology, 21, 92-107.

Karademas, E. C. (2006). Self-efficacy, social support and wellbeing. The mediating role of optimism. Personality and Individual Differences, 40, 1281-1290.

Marcus, D. K. \& Nardone, M. E. (1992). Depression and interpersonal rejection. Clinical Psychology Review, 12, 433-449.

Montoya, R. \& Horton, R. S. (2004). On the importance of cognitive evaluation as a determinant of interpersonal attraction. Journal of Personality and Social Psychology, 86, 696712.

Paulhus, D. L. (1998). Interpersonal and intrapsychic adaptiveness of trait self-enhancement: A mixed blessing? Journal of Personality and Social Psychology, 74, 1197-1208. 
Peterson, C. \& Bossio, L. M. (2001). Optimism and physical well-being. In E. C. Chang (Ed.), Optimism \& pessimism: Implications for theory, research, and practice (pp. 127145). Washington: American Psychological Association.

Robinson, M. D., Johnson, J. T. \& Shields, S. A. (1995). On the advantages of modesty: The benefits of a balanced self-presentation. Communication Research, 22, 575-591.

Scheier, M. F. \& Carver, C. S. (1987). Dispositional optimism and physical well-being: The influence of generalized outcome expectancies on health. Journal of Personality, 55, 169-210.

Scheier, M. F., Carver, C. S. \& Bridges, M. W. (2001). Optimism, pessimism, and psychological well-being. In E. d. C. Chang (Ed.), Optimism \& pessimism: Implications for theory, research, and practice (pp. 189-216). Washington: American Psychological Association.

Schwarzer, R. \& Weiner, B. (1990). Die Wirkung von Kontrollierbarkeit und Bewältigungsverhalten auf Emotionen und soziale Unterstützung. Zeitschrift für Sozialpsychologie, 21, 118-125.

Segerstrom, S. C. (2001). Optimism, goal conflict, and stressorrelated immune change. Journal of Behavioral Medicine, 24, 441-467.

Shen, B. J., McCreary, C. P. \& Myers, H. F. (2004). Independent and Mediated Contributions of Personality, Coping, Social Support, and Depressive Symptoms to Physical Functioning Outcome Among Patients in Cardiac Rehabilitation. Journal of Behavioral Medicine, 27, 39-62.

Silver, R. C., Wortman, C. B. \& Crofton, C. (1990). The role of coping in support provision: The self-presentational dilemma of victims of life crises. In B. R. Sarason, I. G. Sarason \& G. R. Pierce (Eds.), Social support: An interactional view (pp. 397-426). Oxford: UK Wiley \& Sons.

Symister, P. \& Friend, R. (2003). The influence of social support and problematic support on optimism and depression in chronic illness: A prospective study evaluating self-esteem as a mediator. Health Psychology, 22, 123-129.

Taylor, S. E., Lerner, J. S., Sherman, D. K., Sage, R. M. \& McDowell, N. K. (2003). Portrait of the self-enhancer: Well adjusted and well liked or maladjusted and friendless? Journal of Personality and Social Psychology, 84, 165-176.

Trunzo, J. J. \& Pinto, B. M. (2003). Social support as a mediator of optimism and distress in breast cancer survivors. Journal of Consulting and Clinical Psychology, 71, 805-811.

Vollmann, M., Renner, B. \& Weber, H. (2007). Optimism and social support: The providers' perspective. Journal of Positive Psychology, 2, 205-215.

Weber, H. (2003). Breaking the rules: Personal and social responses to coping norm-violations. Anxiety, Stress and Coping: An International Journal, 16, 133-153.

Weber, H., Vollmann, M. \& Renner, B. (2007). The spirited, the observant, and the disheartened: Social concepts of optimism, realism, and pessimism. Journal of Personality, 75, 169-197.

Winer, D. L., Bonner, T. O., Blaney, P. H. \& Murray, E. J. (1981). Depression and social attraction. Motivation and Emotion, 5, 153-166.

Dipl.-Psych. Manja Vollmann

Institut für Psychologie

Universität Greifswald

Franz-Mehring-Straße 47

17487 Greifswald

E-Mail: vollmann@uni-greifswald.de 


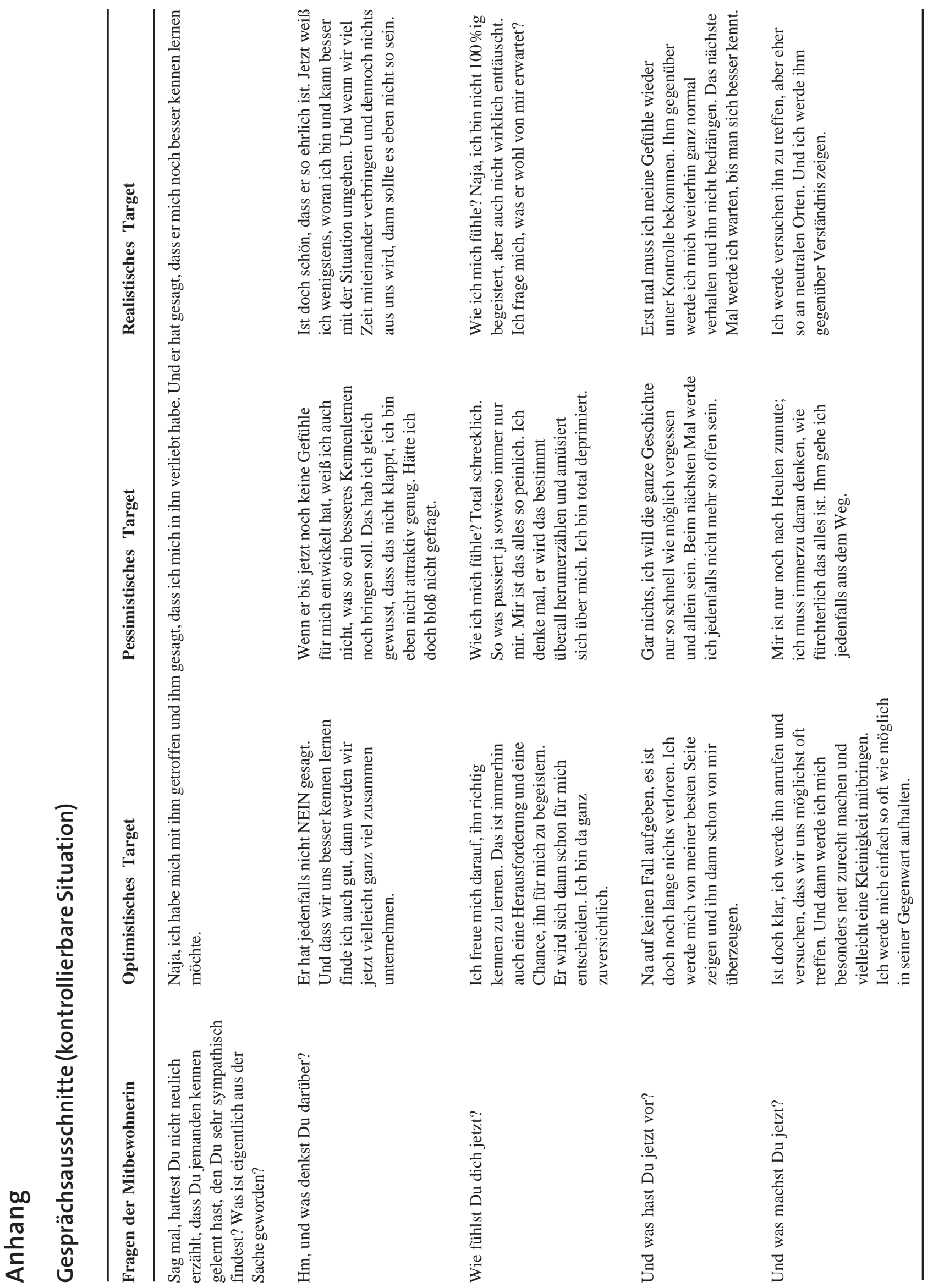

